\title{
General Psychiatry Adolescent anxiety disorders and the developing brain: comparing neuroimaging findings in adolescents and adults
}

\author{
Shuqi Xie, ${ }^{1}$ Xiaochen Zhang (D) , ${ }^{1}$ Wenhong Cheng (D) , ${ }^{2,3}$ Zhi Yang ${ }^{1,4}$
}

To cite: Xie S, Zhang X, Cheng W, et al. Adolescent anxiety disorders and the developing brain: comparing neuroimaging findings in adolescents and adults. General Psychiatry 2021;34:e100411. doi:10.1136/ gpsych-2020-100411

Received 29 September 2020 Accepted 21 June 2021

D) Check for updates

(c) Author(s) (or their employer(s)) 2021. Re-use permitted under CC BY-NC. No commercial re-use. See rights and permissions. Published by BMJ.

'Laboratory of Psychological Health and Imaging, Shanghai Mental Health Center, Shanghai Jiao Tong University School of Medicine, Shanghai, China ${ }^{2}$ Department of Child and Adolescent Psychiatry, Shanghai Mental Health Center, Shanghai Jiao Tong University School of Medicine, Shanghai, China ${ }^{3}$ Department of Psychological Medicine, Shanghai General Hospital, Shanghai Jiao Tong University School of Medicine, Shanghai, China

${ }^{4}$ Institute of Psychological and Behavioral Sciences, Shanghai Jiao Tong University, Shanghai, China

Correspondence to Dr Xiaochen Zhang; xiaochenzhang@smhc.org.cn

\section{ABSTRACT}

Adolescence is the peak period for the incidence of anxiety disorders. Recent findings have revealed the immaturity of neural networks underlying emotional regulation in this population. Brain vulnerability to anxiety in adolescence is related to the unsynchronised development of anxiety-relevant brain functional systems. However, our current knowledge on brain deficits in adolescent anxiety is mainly borrowed from studies on adults. Understanding adolescent-specific brain deficits is essential for developing biomarkers and brain-based therapies targeting adolescent anxiety. This article reviews and compares recent neuroimaging literature on anxietyrelated brain structural and functional deficits between adolescent and adult populations, and proposes a model highlighting the differences between adolescence and adulthood in anxiety-related brain networks. This model emphasises that in adolescence the emotional control system tends to be hypoactivated, the fear conditioning system is immature, and the reward and stress response systems are hypersensitive. Furthermore, the striatum's functional links to the amygdala and the prefrontal cortex are strengthened, while the link between the prefrontal cortex and the amygdala is weakened in adolescence. This model helps to explain why adolescents are vulnerable to anxiety disorders and provides insights into potential brain-based approaches to intervene in adolescent anxiety disorders.

Anxiety disorders are the most common types of mental illnesses in China, and their incidence has been gradually increasing over the past 30 years. ${ }^{1}$ Anxiety disorders mainly manifest as excessive fear, worry and avoidance that induce severe emotional distress, somatic diseases, and cognitive and behavioural impairments, and in turn damage normal social functioning and negatively affect quality of life. $^{2}$ With high lifetime prevalence and high risk for recurrence, there is still a lack of effective treatments for anxiety disorders. ${ }^{3}$ This may be related to the unclarity of the psychopathological mechanisms underlying emotional dysfunction. ${ }^{4}$
Anxiety disorders have an early onset; their incidence among children and adolescents is higher than that among other age groups. ${ }^{5}$ This may be related to the developmental trajectory of the adolescent brain. The brain structure changes significantly from childhood to adolescence in terms of myelination and synapse pruning. ${ }^{6}$ Hormones of puberty, together with pressures from the external environment, reshape the central neural system. These developmental processes and abnormalities may trigger and/or mediate the onset and progression of anxiety disorders in adolescence. In this article, we would review anxiety-relevant abnormalities in the developing brain and attempt to propose a psychopathological model of neural systems underlying anxiety disorders for adolescence, emphasising the differences between adolescents and adults.

\section{BRAIN STRUCTURES ASSOCIATED WITH ANXIETY DISORDERS}

Previous studies have shown that the amygdala, prefrontal cortex, bed nucleus of the stria terminalis (BNST), hippocampus, striatum, anterior insula, anterior cingulate cortex and hypothalamus were closely related to anxiety disorders. ${ }^{7-9}$ For instance, the activation in the hippocampus, amygdala and anterior insula during negative emotion processing was enhanced in patients with anxiety disorders. ${ }^{10}$ In addition, abnormal thalamic volume is an indicator of social anxiety, especially in children and adolescents. $^{11}$ The neural networks that regulate social vigilance mainly comprise the bed nucleus of the stria terminalis and prefrontal cortex. $^{12}$ Patients with anxiety disorders usually show behavioural avoidance, and the avoidance neural network includes the dorsal anterior cingulate cortex, anterior insula, 
sublenticular extended amygdala and temporal pole. Notably, the functional connectivity between the amygdala and the anterior insula is associated with the degree of avoidance. ${ }^{13}$ These structures, which are closely related to anxiety, may play unique roles in the development of cognitive and emotional capabilities among adolescents.

\section{Amygdala}

Previous literature supports that the amygdala is related to fear learning and that its pathological increase in volume is a sign of anxiety disorders. ${ }^{14}$ As has been shown, scary scenes elicit stronger activations in the amygdala among young people with anxiety disorders than healthy controls. ${ }^{15}$ Within the amygdala, the basolateral amygdala participates in processing emotional and somatic responses of anxiety by integrating and transmitting sensory information. ${ }^{16}$ Various projections from the basolateral amygdala regulate anxiety-like behaviours after fear extinction. ${ }^{9}$ Amygdala-based neural networks have been observed to be interrupted among adolescents with anxiety disorders. ${ }^{17}$

\section{Prefrontal cortex}

The prefrontal cortex contributes to fear conditioning and anxiety symptoms. The activation of the medial prefrontal cortex (mPFC) in adolescents is weakened during extinction recall. ${ }^{18}$ The activation of the mPFC of anxious adolescents, however, is stronger than that of the healthy group during negative emotion processing. ${ }^{19}$ Trait anxiety is positively correlated with the activation of the right prefrontal cortex. ${ }^{20}$ High levels of anxiety symptoms are related to delayed development of the neural circuits including the prefrontal cortex in children and adolescents. $^{21}$

\section{Hippocampus}

The hippocampus is a crucial area for encoding and retrieving spatial and contextual memories, and its integrity underlies fear conditioning, which is essential for anxiety and defensive behaviours. ${ }^{22}$ Damages to the hippocampus in non-human primates impair the expression and extinction of fear and thus alter anxiety-related avoidance behaviours. ${ }^{23}$ Similarly, in rodent models, lesions in the ventral hippocampus alter anxiety-like behaviours. ${ }^{24}$ In adolescents, decreased hippocampus volume is one of the risk factors for anxiety disorders. ${ }^{25}$

\section{Bed nucleus of the stria terminalis}

The BNST is the extended part of the amygdala that participates in stress response and anxiety maintenance and is responsible for chronic anxiety. ${ }^{26}$ The BNST mediates the regulation of anxiety by neural circuits underlying substance abuse. For instance, its plasticity alters in adolescent nicotine users and may in turn produce anxiety-like behaviours. ${ }^{27}$ In addition, it also mediates the regulation of anxiety by the hypothalamic-pituitary-adrenal (HPA) axis, whose imbalance may result in anxiety disorders. ${ }^{28}$

\section{Striatum}

The striatum includes the nucleus accumbens, caudate nucleus and putamen and is usually considered as a part of the reward circuit. The striatum develops in early adolescence. ${ }^{29}$ The striatum contributes to anxiety symptoms and anxiety-related bias in emotional, motivational and attentional processes, since it has vital contributions to these processes, and striatum-based functional connectivity differs between anxious adolescents and healthy adolescents. $^{730}$

\section{Hypothalamus}

The hypothalamus is a critical structure involved in the anxiety circuit. It is also a central part of the HPA axis responsible for regulating emotions, defensive behaviour, aggression and stress responses. The hypothalamus actively controls aggressive and anxious behaviours by influencing hormone synthesis in adolescence. ${ }^{31}$ Factors that affect HPA axis activities include circadian rhythm disturbances, stress and caffeine, particularly during adolescence. $^{32}$ The dysregulation of HPA axis activities during adolescence increases the risk of developing anxiety disorders.

\section{NEURAL NETWORKS UNDERLYING ADOLESCENT ANXIETY}

Anxiety disorders are not due to deficits of a single brain structure. Instead, there are plenty of studies suggesting anxiety-related neural networks. This section reviews previous findings that link deficits in neural networks to anxiety, especially in adolescents.

Anxiety disorders have abnormalities in many aspects of psychological processes, such as cognitive control, fear conditioning, uncertainty anticipation, motivation bias and stress regulation. Regarding cognitive control, anxiety disorders are characterised by dysfunctional cognitive control of the projection from the prefrontal cortex to the amygdala. ${ }^{13}$ Patients with anxiety disorders show alterations in the fear condition, including abnormalities in the network involving the ventral hippocampus, basolateral amygdala and mPFC. ${ }^{22}$ Regarding uncertainty anticipation, neural pathways involving the BNST mediate the over-reaction to uncertain anticipations in patients with anxiety disorders. ${ }^{33}$ As for motivation, the striatum is associated with unbalanced reward function in anxiety disorders, and the failed regulation of the striatum, amygdala and prefrontal cortex is an important neural underpinning of anxiety disorders. ${ }^{34}$ Regarding stress, abnormal recruitment of the hypothalamus, amygdala and prefrontal cortex is associated with the failure of stress regulation. Based on the mentioned associations, we assume that psychopathological symptoms of anxiety disorders in adolescence may be underlain with abnormalities in brain structures relevant to cognitive control, fear conditioning, uncertainty anticipation, motivational processing and stress regulation.

Figure 1 summarises previous findings regarding the deficits of neural networks underlying anxiety disorders, characterised by the five components. We will further review 

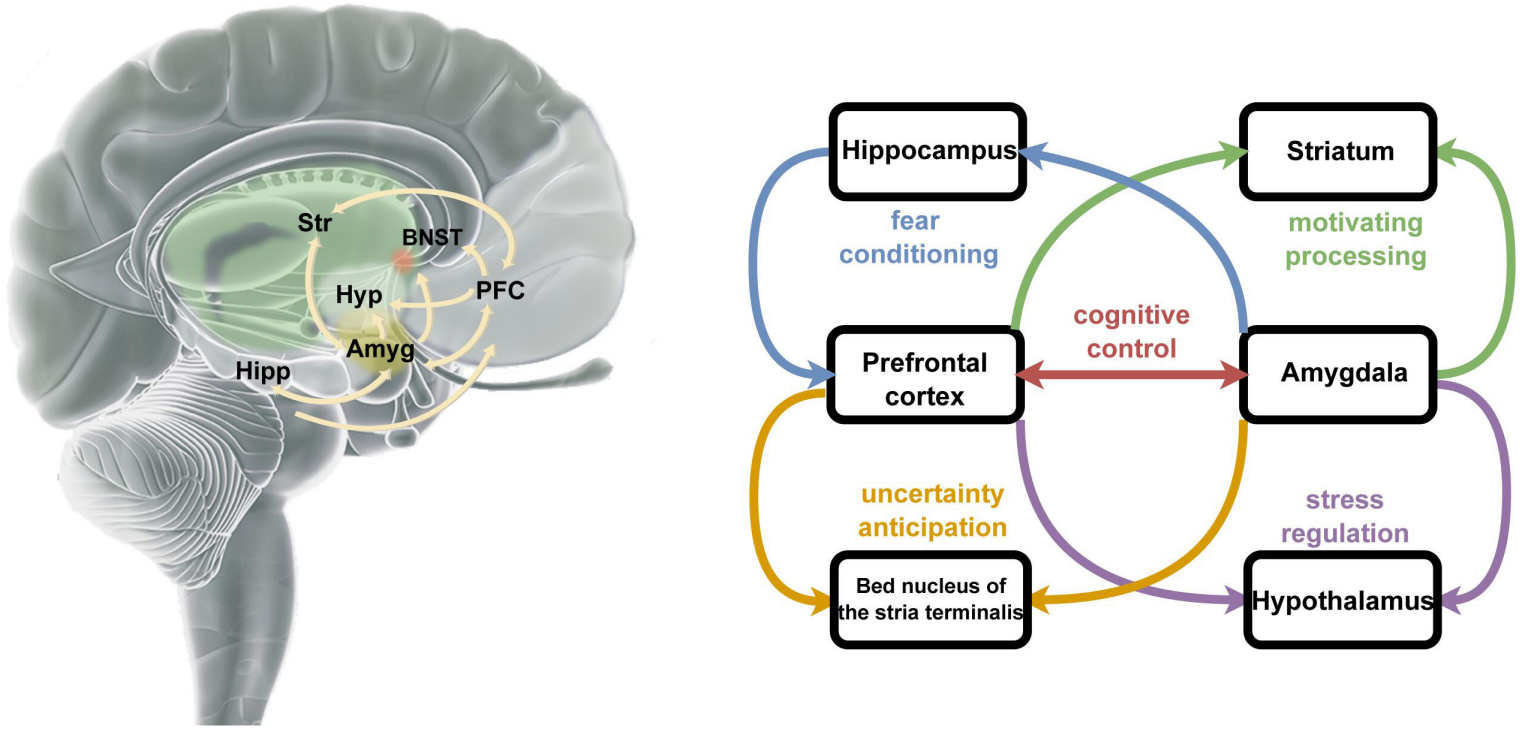

Figure 1 Schematic presentation of anxiety-related functional systems and brain structures. The arrows indicate the neural projections between two brain regions. The pathological interactions of the six critical regions in the anxiety network bring about functional abnormality for patients with anxiety disorders. Amyg, Amygdala; BNST, Bed nucleus of the stria terminalis; Hipp, Hippocampus; Hyp, Hypothalamus; PFC, Prefrontal cortex; Str, Striatum.

the findings relevant to these components in the following subsections.

\section{Cognitive control: amygdala and mPFC}

The neural circuit between the mPFC and the amygdala is closely related to cognitive control. The amygdala projects to different areas of the mPFC; specifically, basal amygdala neurons project to the prelimbic and the infralimbic subdivision of the mPFC, completing the fear expression and extinction. ${ }^{35}$ The activation of this pathway corresponds to an increase in individual anxiety-like behaviours and a decrease in social interaction. ${ }^{36}$ The mPFC integrates inputs from various areas and projects them back to the amygdala to achieve top-down inhibitory control. The number of basal amygdala neurons projecting to the mPFC tends to become stable during adolescence. ${ }^{4}$ In adolescence, the maturation of the mPFC is later than that of the amygdala, and the ability of top-down regulation is immature and weak. ${ }^{37}$ Hence, insufficient inhibition of the amygdala neurons may provide a potential neural basis for adolescent anxiety disorders.

Fear conditioning: ventral hippocampus, basolateral amygdala and $\mathrm{MPFC}$

Deficiency in fear extinction is one of the characteristics of anxiety disorders. The ventral hippocampus, basolateral amygdala and the mPFC form an interconnected circuit that plays a vital role in fear learning and extinction. $^{38} 39$ The $\mathrm{mPFC}$ receives outputs from the ventral hippocampus. Glutamatergic inputs from the ventral hippocampus enhance the plasticity of $\mathrm{mPFC}$ neurons and promote maturation. ${ }^{40}$ When the ventral hippocampus projects to the mPFC, neurons synchronise to the theta frequency ( 4 to $12 \mathrm{~Hz}$ ), forming and maintaining anxiety-like behaviours. ${ }^{39} 41$ In contrast, inhibiting the projections from the ventral hippocampus to the mPFC reduces the synchrony of the theta frequency and reduces the probability of anxiety disorders. $^{39}$

The ventral hippocampus and the basolateral amygdala rely on each other coding fear-related memories. ${ }^{42}$ Glutamatergic inputs from the basolateral amygdala to the ventral hippocampus pyramidal neurons increase individual anxiety, while inhibition of this projection reduces anxiety-related behaviours. ${ }^{43}$ The pathway projected from the basolateral amygdala to the ventral hippocampus regulates social interactions as well. ${ }^{42}$ The dual function of this pathway in modulating anxiety and social behaviours possibly explains the high comorbidity rate of anxiety disorders and autism spectrum disorders. ${ }^{44}$ The projections from the ventromedial prefrontal cortex to the amygdala inhibit fear expression, and this process is modulated by the hippocampus. ${ }^{45}$

\section{Uncertainty anticipation: BNST, amygdala and prefrontal cortex}

The neural networks that include the BNST, amygdala and prefrontal cortex participate in the anticipation of uncertain threats. ${ }^{3346}$ There is a functional separation between the basolateral amygdala and the BNST. The basolateral amygdala mediates immediate responses to threats and is related to panic disorder and specific phobia in anxiety disorders. The BNST, in contrast, mediates sustained responses to unpredictable threat information and is related to generalised anxiety disorder and post-traumatic stress disorder. ${ }^{48}$ The excessive and 
persistent anxiety response of patients with anxiety disorders to uncertain information may be underlain with neural projections from the basolateral amygdala to the BNST. $^{33}$ The BNST receives the inputs from the mPFC. ${ }^{7}$ The nerve connection between the MPFC and the BNST has a modulating effect on anxiety. ${ }^{49}$

\section{Motivation processing: striatum, amygdala and prefrontal cortex}

The nucleus accumbens of the striatum evaluates stimuli in terms of motivational values, and the dorsal striatum integrates and transmits information to the prefrontal and motor cortices. ${ }^{50}$ Reward representations are available to the ventral striatum that participates in forming motivational and goal-oriented behaviours. The amygdala actively regulates the striatum. The direct projection from the amygdala to the striatum supports 'fight or flight' motor responses, as well as avoidance learning. ${ }^{33}$ In addition, the ventral striatum participates in emotion and motivation processing, driving action outputs from the basal ganglia. ${ }^{51}$ The projection from the prefrontal cortex to the striatum contributes to cognitive functions, such as decision-making. ${ }^{52}$ The mPFC achieves a balance between anxiety-like and motivated behaviours by the inputs to the amygdala and the striatum. ${ }^{53}$

\section{Stress regulation: hypothalamus, amygdala and prefrontal cortex}

The hypothalamus is sensitive to stressors and plays a regulatory role. Under stressful conditions, amygdalato-hypothalamic outputs maintain anxiety behaviours. ${ }^{54}$ The neurotransmissions from the hypothalamus to the amygdala build fear expression and generalisation, and the deactivation of hypothalamic orexin neurons alleviates excessive fear. ${ }^{55}$ The direct projection from the prefrontal cortex to the hypothalamus involves regulating emotional stress, emotional arousal and control of aggressive behaviours. ${ }^{56}$ In anxiety-inducing situations, the prefrontal cortex inputs dopamine to the amygdala, which further excites the hypothalamic neurons and initiates the HPA axis, triggering a somatic response of sympathetic excitation. ${ }^{57}$ In patients with anxiety disorders, overactivation of the amygdala leads to overexpression of corticotropin-releasing factors, promoting HPA axis hyperactivity and manifesting as an over-reaction to stress. ${ }^{58}$

\section{Differences in brain networks across subtypes of anxiety disorders}

Morphologically, patients with social anxiety disorder (SAD) have a larger grey matter volume in the dorsal striatum. ${ }^{59}$ They have reduced frontal lobe volume and increased amygdala volume relative to healthy controls. ${ }^{6061}$ Patients with generalised anxiety disorder (GAD) have reduced ventromedial prefrontal cortex volume and hypothalamus volume. ${ }^{62-64}$ Patients with panic disorder (PD) have smaller grey matter volumes in the amygdala, the hippocampus, the prefrontal cortex and the bilateral striatum. ${ }^{6566}$

During emotion-related tasks, the amygdala and the parahippocampal gyrus are overactive in patients with $\mathrm{SAD}$, with enhanced functional connectivity between the amygdala and the prefrontal cortex.$^{6768}$ In contrast, patients with GAD have insufficient activation in the prefrontal cortex and weak functional connectivity between the amygdala and the prefrontal cortex. ${ }^{69}$ In gambling games, BNST activity is increased while amygdala activity is suppressed in patients with GAD, where anxiety experience is triggered by high uncertainty. ${ }^{70}$ In fear conditioning, patients with PD have hyperactivation in the hypothalamus and abnormal recruitments of the hippocampal-prefronto-amygdala network with hippocampal excitation, manifesting as excessive fear learning. ${ }^{67} 71$

Brain network differences exist in different subtypes of anxiety disorders and are reflected differentially in various brain structures. Notably, current treatments for anxiety disorders also have different efficacy for different subtypes; for example, cognitive-behavioural therapy to treat patients with SAD tends to be less effective for other anxiety disorders. ${ }^{61}$ Possible neural underpinnings are that the aberrance patterns of the anxiety network are different for different subtypes of anxiety disorders: hypersensitivity to emotional stimuli in patients with SAD, inadequate top-down control in patients with GAD and excessive fear learning in patients with PD. With future verifications of this hypothesis, changes in the anxiety network may help to predict the efficacy of treatments. ${ }^{72}$

\section{UNIQUE FEATURES OF THE ADOLESCENT ANXIETY-RELATED NETWORKS}

Based on the above review, we further propose that the imbalance in the developmental progress of these five systems during adolescence provides an essential basis for the high incidence of anxiety disorders during this period. From adolescence to adulthood, the neural connections in anxiety neural networks change dramatically. For example, the intensity of connections between subcortical structures decreases with age ${ }^{73}$; the long and extensive cortical connections gradually dominate and the regulation to subcortical regions is enhanced, forming the neural basis for the gradual improvement in emotional processing. ${ }^{74}$ As shown in figure 2 , the anxiety network of adolescents and adults differs in regional activations and structural and functional connectivity within the anxiety network.

\section{Insufficient cognitive control in adolescence}

During emotion processing, amygdala activation shows a non-monotonic change with age, with reduced amygdala activation more in adults than in adolescents. ${ }^{75}$ During adolescence, the activation of the prefrontal cortex in performing cognitive tasks becomes more focal with age, and adults show stronger prefrontal activation than 


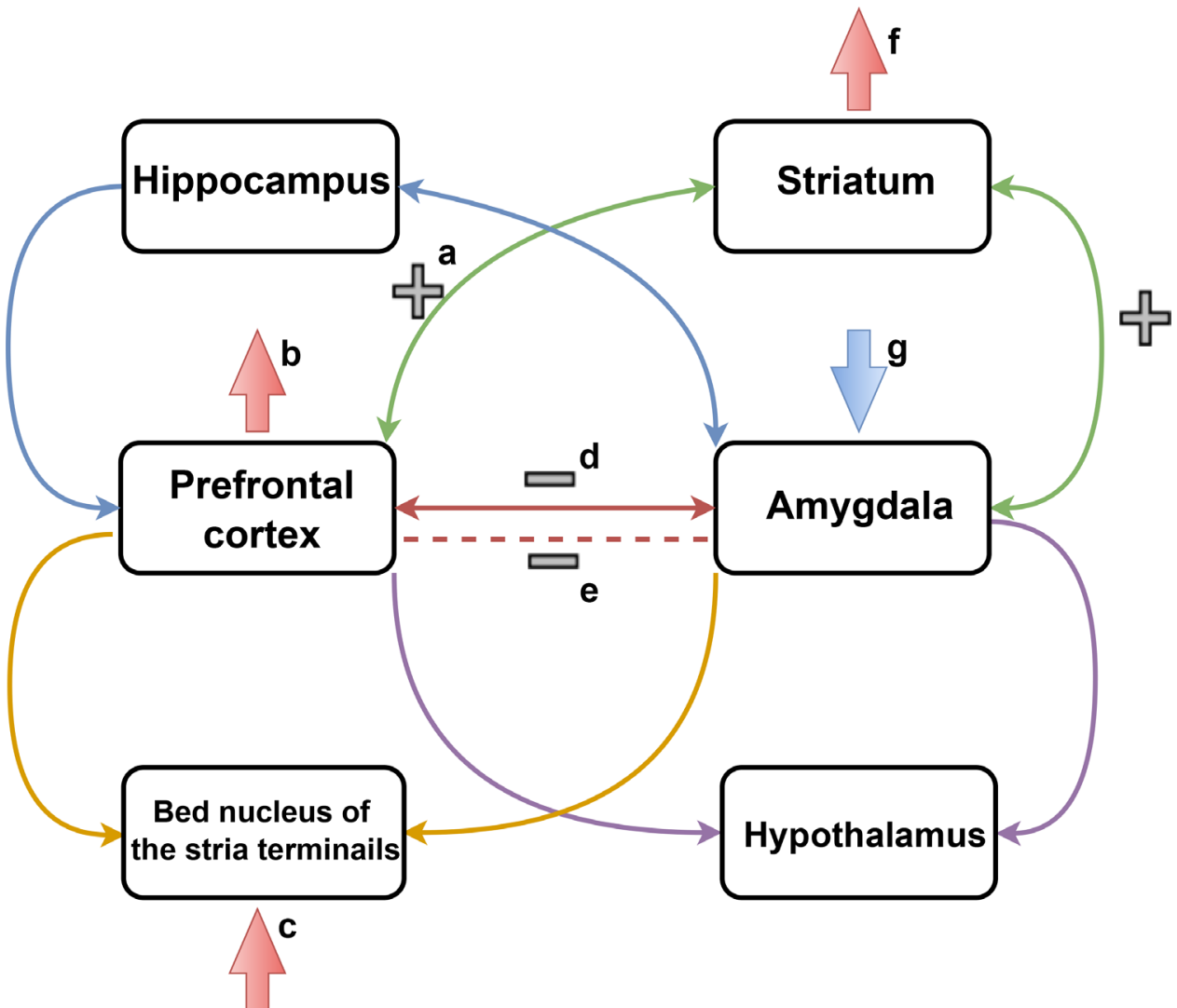

Figure 2 Schematic of the differences between adolescence and adulthood anxiety networks. ' $\uparrow$ ' or ' $\downarrow$ ' indicates that the activation of the marked brain region in adolescents is higher or lower than that in adults; '+' or '-' indicates that the connection of this pathway is stronger or weaker in adolescents compared with that of adults. Solid lines indicate functional connections, and dashed lines indicate structural connections. a, DePasque \& Galvan, $2017 ;{ }^{98}$ b, Casey et al, $2008 ;{ }^{76} \mathrm{c}$, Saalfield \& Spear, 2019; ${ }^{92}$ d, Pattwell et al, 2016; ${ }^{88}$ e, Lebel \& Beaulieu, 2011:78 f, Galvan, 2010; ${ }^{95} \mathrm{~g}$, Hare et al, $2008 ;{ }^{75} \mathrm{~h}$, Heller et al, $2016 .{ }^{74}$

adolescents. ${ }^{76}$ Compared with adolescents, adults are able to exert cognitive control of the prefrontal cortex in anxious situations and are less affected by emotional interference. $^{75}$

Functional and structural connections between the prefrontal cortex and the amygdala are reorganised during adolescent development. ${ }^{77}$ The structural connectivity between the amygdala and the prefrontal cortex increases, in terms of fractional anisotropy, with age. ${ }^{78}$ Importantly, reduced integrity of this structural connection in adults and adolescents is associated with anxiety disorders. ${ }^{79}$ In addition, delayed maturation of amygdalaprefrontal connections during adolescence may be one of the neural bases for abnormalities in emotional behaviours. ${ }^{80}$ The number of dendritic spines in $\mathrm{mPFC}$ neurons and basolateral amygdala neurons is larger in adolescence than in adulthood due to dendritic remodelling and pruning. However, the later the dendritic remodelling and pruning are completed, the higher the risk of developing anxiety disorders. ${ }^{81}$ The signalling endocannabinoid that impacts cognitive control in the prefrontal cortex is enhanced in adolescence, while weaker signalling is found in adolescents with anxiety disorders. ${ }^{82}$

The functional connectivity between the amygdala and the prefrontal cortex during emotion-related tasks shifts from positive to negative from early childhood to adolescence, which may be related to the development of the capacity to deal with separation anxiety. ${ }^{83}$ From adolescence to adulthood, the amygdala-prefrontal functional connectivity shifts from negative to more strongly negative, reflecting the increased regulation of emotions for adults. ${ }^{84}$ Therefore, reduced functional connectivity between the amygdala and the prefrontal cortex indicates failure of top-down control in the prefrontal cortex, which is a potential pathological factor for anxiety disorders in both adolescents and adults. ${ }^{85} 86$

\section{Ineffective fear extinction in adolescence}

Hippocampal volume increases and then decreases from adolescence to adulthood. ${ }^{87}$ During adolescence, the structural connections between the amygdala and the mPFC develop, which is related to fear conditioning. ${ }^{88}$ Relative to adults, even mentally healthy adolescents are ineffective at fear extinction. ${ }^{89}$ Negative functional connectivity in adolescents between the prefrontal cortex and the hippocampus and between the prefrontal cortex and the amygdala is related to deficits in extinction recall and with state anxiety. ${ }^{45}$ In addition, the ventral hippocampus has an ineffective inhibitory effect on the functional connectivity between the basolateral lateral amygdala and the mPFC in adolescents, thereby impacting fear extinction. Disruption of these processes 
may lead to pathological emotional responses in psychiatric disorders. ${ }^{40}$ For adolescents, excessive functional connectivity between the prefrontal cortex, the amygdala and the hippocampus is associated with a higher risk of anxiety disorders. For adults, the lack of hippocampal inhibition of the prefrontal cortex, which is common in typically developing adolescents, may increase the risk of mood disorders in adults. ${ }^{90}$

\section{Increased response to anticipating uncertainty}

The BNST is activated in an environment with unpredictable threats. ${ }^{91}$ The reaction of the BNST in adolescents tends to be stronger than in adults. ${ }^{92}$ This may partially explain the high susceptibility of adolescents to anxiety disorders. After all, during adolescence, hyperactivation of the BNST and the basolateral amygdala accompanies increment of anxiety-like behaviours in individuals. ${ }^{93}$

For adults with social anxiety disorder, the BNST responds more strongly to unpredictable emotional faces. This may be a consequence of weakened functional connectivity between the BNST and the amygdala. ${ }^{91}$ The mPFC promotes the activity of the BNST and enhances negative emotions and behaviours. ${ }^{49}$ The functional connectivity between the BNST and the prefrontal cortex is negatively correlated with anxiety severity in patients with anxiety disorders but positively correlated in healthy controls. ${ }^{94}$ However, the relationship between the development of the BNST and anxiety disorders in adolescents, as well as the neural connections of the BNST and their functions during adolescence, is underdocumented and needs further investigation.

\section{Motivational processing bias in adolescence}

In reward tasks, peak striatum activation occurs in adolescence relative to childhood and adulthood. Adolescents are more sensitive to rewards as the reward stimuli of the same intensity elicit stronger ventral striatal activation in adolescents. ${ }^{95}$ In contrast, patients with anxiety disorders, who tend to be risk-avoidant, show overactivations at the striatum, which may be one of the neural bases of anxiety disorders. ${ }^{796}$

The functional connectivity between the amygdala and the striatum in response to emotional cues is declining during development, and is associated with enhanced cognitive control. ${ }^{74} 97$ Similarly, the functional connectivity between the prefrontal cortex and the striatum also declines with age. ${ }^{98}$ Adolescents with GAD show increased functional connectivity between the striatum and the amygdala, accompanying higher sensitivity to reward-related stimuli. ${ }^{99}$ Patients with anxiety disorders also show altered prefrontal-striatal functional connectivity. ${ }^{50}$ The prefrontal-striatum connectivity in adults with anxiety disorders is increased, ${ }^{100}$ whereas that in adolescents with anxiety disorders may decrease in response to unexpected positive feedback. ${ }^{30}$ The abnormal development of dopaminergic neurons in the amygdala, the prefrontal cortex, the hippocampus and the striatum during adolescence alters one's exposure to hormonal influences, which may increase the risk of mental illness. ${ }^{101}$

\section{Hypersensitivity to stress in adolescence}

For adolescents, experiencing stress leads to overexpression of corticotropin-releasing factor receptor 1 in the hypothalamus, the amygdala and the prefrontal cortex, causing abnormalities in the HPA axis that will persist into adulthood. ${ }^{102}$ Significantly increased responses to stress in adolescents compared with adults, as evidenced by prolonged hormonal exposure, are possibly responsible for the increased vulnerability to psychiatric disorders seen during adolescence. ${ }^{103}$

\section{CONCLUSIONS}

Adolescents are immature in terms of emotional regulation. During adolescence, the emotional control system tends to be hypoactivated, echoing the insufficient cognitive control in adolescents; the fear conditioning system is immature, echoing the ineffective fear extinction; and the reward system and the stress response system are both hypersensitive, echoing the biased motivational processing and the aberrant stress regulation when facing circumstances with potential threats, respectively. These unique features of adolescent brains may partially explain the vulnerability of adolescents to anxiety disorders.

While there has been a large body of literature on the differences of separate anxiety-related functional circuits between adolescents and adults, studies on whether and how the separate functional circuits integrate to represent the vulnerability of adolescents to anxiety are still rare. The anatomical connections and the functional relevance of the five circuits reviewed in the paper are apparent, suggesting the high likelihood of interactions among the functional circuits. Future research should develop and apply advanced methodologies, such as functional network connectivity and multivariate pattern analyses, to reveal the relationships between the interactions of the functional circuits and the risk of adolescent anxiety.

Furthermore, the adolescent-specific brain abnormalities have some clinical implications for adolescent anxiety. The neuroimaging markers for diagnosis and early detection of anxiety in adolescents may differ from those found in adult studies and therefore require further investigations and verifications in the adolescent population. In addition, brain abnormalities in adolescents suggest potential targets for brain-based therapies such as brain modulations. The links between different brain systems and various symptoms and subtypes have implications for individualised brain-based treatments.

This review focused on comparing neuroimaging findings on the anxiety-related brain circuits between adolescents and adults, providing a macro-scale model connecting the adolescent-specific brain features with vulnerability to anxiety. It should be noted that 
the essential drives of the functional circuits, such as neuronal properties and neural transmitters, are the bases of the macro-scale observations. Future theoretical and experimental work is expected to reveal theories and findings regarding adolescents' vulnerability to anxiety.

Contributors SX performed the literature search and drafted the manuscript. XZ, WC and ZY designed the review and edited the manuscript. All authors read and approved the final version of the manuscript.

Funding This work was supported by the National Key R\&D Program of China (2018YFC2001600); National Natural Science Foundation of China (81971682, 81571756, 81270023); Natural Science Foundation of Shanghai (20ZR1472800); Project of Shanghai Children's Health Service Capacity Construction (GDEK201702); Shanghai Sailing Programme (2OYF1441900); Shanghai Municipal Commission of Education-Gaofeng Clinical Medicine Grant Support (20171929); Hundred-Talent Fund from Shanghai Municipal Commission of Health (2018BR17); Shanghai Mental Health Center Clinical Research Center (CRC2018DSJ01-5; CRC2019ZD04); Research Funds from Shanghai Mental Health Center (13dz2260500); Institute-level Projects of Shanghai Mental Health Center (2020-YJ03, 2018-YJ-02, 2018-YJ-03); Qihang Foundation of Shanghai Mental Health Center (2019-QH-02); and Shanghai Jiao Tong University School of Medicine Child Developing Brain Research Centre.

Competing interests None declared.

Patient consent for publication Not required.

Provenance and peer review Not commissioned; externally peer reviewed.

Open access This is an open access article distributed in accordance with the Creative Commons Attribution Non Commercial (CC BY-NC 4.0) license, which permits others to distribute, remix, adapt, build upon this work non-commercially, and license their derivative works on different terms, provided the original work is properly cited, appropriate credit is given, any changes made indicated, and the use is non-commercial. See: http://creativecommons.org/licenses/by-nc/4.0/.

\section{ORCID iDs}

Xiaochen Zhang http://orcid.org/0000-0002-1851-0098

Wenhong Cheng http://orcid.org/0000-0003-4469-9442

\section{REFERENCES}

1 Huang $\mathrm{Y}$, Wang $\mathrm{Y}$, Wang $\mathrm{H}$, et al. Prevalence of mental disorders in China: a cross-sectional epidemiological study. Lancet Psychiatry 2019;6:211-24.

2 Duval ER, Javanbakht A, Liberzon I. Neural circuits in anxiety and stress disorders: a focused review. Ther Clin Risk Manag 2015;11:115.

3 Bui E, King F, Melaragno A. Pharmacotherapy of anxiety disorders in the 21st century: a call for novel approaches. Gen Psychiatr 2019;32:e100136.

4 Zimmermann KS, Richardson R, Baker KD. Maturational changes in prefrontal and amygdala circuits in adolescence: implications for understanding fear inhibition during a vulnerable period of development. Brain Sci 2019;9:65.

5 Powers A, Casey BJ. The adolescent brain and the emergence and peak of psychopathology. J Infant Child Adolesc Psychother 2015;14:3-15

6 Konrad K, Firk C, Uhlhaas PJ. Brain development during adolescence. Dtsch Aerzteblatt Online 2013;110:425-31.

7 Lago T, Davis A, Grillon C, et al. Striatum on the anxiety map: small detours into adolescence. Brain Res 2017;1654:177-84.

8 Calhoon GG, Tye KM. Resolving the neural circuits of anxiety. Nat Neurosci 2015;18:1394-404

9 Tovote P, Fadok JP, Lüthi A. Neuronal circuits for fear and anxiety. Nat Rev Neurosci 2015;16:317-31.

10 Carlisi CO, Hilbert K, Guyer AE, et al. Sleep-amount differentially affects fear-processing neural circuitry in pediatric anxiety: a preliminary fMRI investigation. Cogn Affect Behav Neurosci 2017;17:1098-113.

11 Zhang Y, Liu W, Lebowitz ER, et al. Abnormal asymmetry of thalamic volume moderates stress from parents and anxiety symptoms in children and adolescents with social anxiety disorder. Neuropharmacology 2020;180:108301.
12 Wright EC, Hostinar CE, Trainor BC. Anxious to see you: neuroendocrine mechanisms of social vigilance and anxiety during adolescence. Eur J Neurosci 2019:1-14.

13 Williams LM. Precision psychiatry: a neural circuit taxonomy for depression and anxiety. Lancet Psychiatry 2016;3:472-80.

14 Albaugh MD, Nguyen T-V, Ducharme S, et al. Age-Related volumetric change of limbic structures and subclinical anxious/ depressed symptomatology in typically developing children and adolescents. Biol Psychol 2017;124:133-40.

15 Beesdo K, Lau JYF, Guyer AE, et al. Common and distinct amygdala-function perturbations in depressed vs anxious adolescents. Arch Gen Psychiatry 2009;66:275-85.

16 Varlinskaya El, Johnson JM, Przybysz KR, et al. Adolescent forced swim stress increases social anxiety-like behaviors and alters kappa opioid receptor function in the basolateral amygdala of male rats. Prog Neuropsychopharmacol Biol Psychiatry 2020;98:109812.

17 Roy AK, Fudge JL, Kelly C, et al. Intrinsic functional connectivity of amygdala-based networks in adolescent generalized anxiety disorder. J Am Acad Child Adolesc Psychiatry 2013;52:290-9.

18 Morriss J, Christakou A, van Reekum CM. Multimodal evidence for delayed threat extinction learning in adolescence and young adulthood. Sci Rep 2019;9:1-10.

19 Lau JYF, Waters AM. Annual research review: an expanded account of information-processing mechanisms in risk for child and adolescent anxiety and depression. $J$ Child Psychol Psychiatry 2017;58:387-407.

20 Telzer EH, Mogg K, Bradley BP, et al. Relationship between trait anxiety, prefrontal cortex, and attention bias to angry faces in children and adolescents. Biol Psychol 2008;79:216-22.

$21 \mathrm{Liu}$ Z, Hu Y, Zhang Y, et al. Altered gray matter volume and structural co-variance in adolescents with social anxiety disorder: evidence for a delayed and unsynchronized development of the fronto-limbic system. Psychol Med 2020:1-10.

22 Giustino TF, Maren S. The role of the medial prefrontal cortex in the conditioning and extinction of fear. Front Behav Neurosci 2015;9:1-20.

23 Machado CJ, Bachevalier J. The impact of selective amygdala, orbital frontal cortex, or hippocampal formation lesions on established social relationships in rhesus monkeys (Macaca mulatta). Behav Neurosci 2006:120:761-86.

24 Trivedi MA, Coover GD. Lesions of the ventral hippocampus, but not the dorsal hippocampus, impair conditioned fear expression and inhibitory avoidance on the elevated T-maze. Neurobiol Learn Mem 2004;81:172-84.

25 Koolschijn PCMP, van IJzendoorn MH, Bakermans-Kranenburg MJ, et al. Hippocampal volume and internalizing behavior problems in adolescence. Eur Neuropsychopharmacol 2013;23:622-8.

26 Robinson OJ, Pike AC, Cornwell B, et al. The translational neural circuitry of anxiety. $J$ Neurol Neurosurg Psychiatry 2019;90:1353-60.

27 Smith KC, Ehlinger DG, Smith RF. Adolescent nicotine alters dendritic morphology in the bed nucleus of the stria terminalis. Neurosci Lett 2015;590:111-5.

28 Lezak KR, Roelke E, Harris OM, et al. Pituitary adenylate cyclaseactivating polypeptide (PACAP) in the bed nucleus of the stria terminalis (BNST) increases corticosterone in male and female rats. Psychoneuroendocrinology 2014;45:11-20.

29 Casey BJ, Heller AS, Gee DG, et al. Development of the emotional brain. Neurosci Lett 2019;693:29-34.

30 Jarcho JM, Romer AL, Shechner T, et al. Forgetting the best when predicting the worst: preliminary observations on neural circuit function in adolescent social anxiety. Dev Cogn Neurosci 2015;13:21-31.

31 Morrison TR, Ricci LA, Puckett AS, et al. Serotonin type-3 receptors differentially modulate anxiety and aggression during withdrawal from adolescent anabolic steroid exposure. Horm Behav 2020;119:104650.

32 Ma D, Serbin LA, Stack DM. How children's anxiety symptoms impact the functioning of the hypothalamus-pituitary-adrenal axis over time: a cross-lagged panel approach using hierarchical linear modeling. Dev Psychopathol 2019;31:309-23.

33 Grupe DW, Nitschke JB. Uncertainty and anticipation in anxiety: an integrated neurobiological and psychological perspective. Nat Rev Neurosci 2013;14:488-501.

34 Sturm V, Lenartz D, Koulousakis A, et al. The nucleus accumbens: a target for deep brain stimulation in obsessive-compulsive- and anxiety-disorders. J Chem Neuroanat 2003;26:293-9.

35 Senn V, Wolff SBE, Herry C, et al. Long-Range connectivity defines behavioral specificity of amygdala neurons. Neuron 2014:81:428-37. 
36 Felix-Ortiz AC, Burgos-Robles A, Bhagat ND, et al. Bidirectional modulation of anxiety-related and social behaviors by amygdala projections to the medial prefrontal cortex. Neuroscience 2016;321:197-209.

37 Mueller SC, Cromheeke S, Siugzdaite R, et al. Evidence for the triadic model of adolescent brain development: cognitive load and task-relevance of emotion differentially affect adolescents and adults. Dev Cogn Neurosci 2017;26:91-100.

$38 \mathrm{Li} \mathrm{L}$, Gao X, Zhou Q. Absence of fear renewal and functional connections between prefrontal cortex and hippocampus in infant mice. Neurobiol Learn Mem 2018;152:1-9.

39 Padilla-Coreano N, Bolkan SS, Pierce GM, et al. Direct ventral hippocampal-prefrontal input is required for anxiety-related neural activity and behavior. Neuron 2016;89:857-66.

40 Thomases DR, Cass DK, Meyer JD, et al. Early adolescent MK-801 exposure impairs the maturation of ventral hippocampal control of basolateral amygdala drive in the adult prefrontal cortex. $J$ Neurosci 2014;34:9059-66.

41 Daviu N, Bruchas MR, Moghaddam B, et al. Neurobiological links between stress and anxiety. Neurobiol Stress 2019;11:100191.

42 Allsop SA, Vander Weele CM, Wichmann R, et al. Optogenetic insights on the relationship between anxiety-related behaviors and social deficits. Front Behav Neurosci 2014;8:1-14.

43 Felix-Ortiz AC, Beyeler A, Seo C, et al. Bla to vHPC inputs modulate anxiety-related behaviors. Neuron 2013;79:658-64.

44 Felix-Ortiz AC, Tye KM. Amygdala inputs to the ventral hippocampus bidirectionally modulate social behavior. $J$ Neurosci 2014;34:586-95.

45 Ganella DE, Barendse MEA, Kim JH, et al. Prefrontal-amygdala connectivity and state anxiety during fear extinction recall in adolescents. Front Hum Neurosci 2017;11:587.

46 Tanovic E, Gee DG, Joormann J. Intolerance of uncertainty: neural and psychophysiological correlates of the perception of uncertainty as threatening. Clin Psychol Rev 2018;60:87-99.

47 Clauss J. Extending the neurocircuitry of behavioural inhibition: a role for the bed nucleus of the stria terminalis in risk for anxiety disorders. Gen Psychiatr 2019;32:e100137.

48 Avery SN, Clauss JA, Blackford JU. The human BNST: functional role in anxiety and addiction. Neuropsychopharmacology 2016;41:126-41.

49 Motzkin JC, Philippi CL, Oler JA, et al. Ventromedial prefrontal cortex damage alters resting blood flow to the bed nucleus of stria terminalis. Cortex 2015;64:281-8.

50 Dorfman J, Benson B, Farber M, et al. Altered striatal intrinsic functional connectivity in pediatric anxiety. Neuropsychologia 2016;85:159-68.

51 Haber SN, Knutson B. The reward circuit: linking primate anatomy and human imaging. Neuropsychopharmacology 2010;35:4-26.

52 Calhoon GG, O'Donnell P. Closing the gate in the limbic striatum: prefrontal suppression of hippocampal and thalamic inputs. Neuron 2013;78:181-90.

53 Calhoon GG, Tye KM. Resolving the neural circuits of anxiety. Nat Neurosci 2015;18:1394-404.

54 Nordman J, Ma X, Li Z. Traumatic stress induces prolonged aggression increase through synaptic potentiation in the medial amygdala circuits. eNeuro 2020;7. doi:10.1523/ ENEURO.0147-20.2020. [Epub ahead of print: 2307 2020].

55 Soya S, Sakurai T. Orexin as a modulator of fear-related behavior: hypothalamic control of noradrenaline circuit. Brain Res 2020;1731:146037.

56 Biro L, Sipos E, Bruzsik B, et al. Task division within the prefronta cortex: distinct neuron populations selectively control different aspects of aggressive behavior via the hypothalamus. J Neurosci 2018;38:4065-75.

57 Mejia-Carmona GE, Gosselink KL, Pérez-Ishiwara G, et al. Oxidant/ antioxidant effects of chronic exposure to predator odor in prefrontal cortex, amygdala, and hypothalamus. Mol Cell Biochem 2015;406:121-9.

58 Flandreau El, Ressler KJ, Owens MJ, et al. Chronic overexpression of corticotropin-releasing factor from the central amygdala produces HPA axis hyperactivity and behavioral anxiety associated with geneexpression changes in the hippocampus and paraventricular nucleus of the hypothalamus. Psychoneuroendocrinology 2012;37:27-38.

59 Bas-Hoogendam JM, van Steenbergen H, Nienke Pannekoek $\mathrm{J}$, et al. Voxel-Based morphometry multi-center mega-analysis of brain structure in social anxiety disorder. Neuroimage Clin 2017;16:678-88.

60 Salokangas RKR, Hietala J, Armio RL, et al. Effect of childhood physical abuse on social anxiety is mediated via reduced frontal lobe and amygdala-hippocampus complex volume in adult clinical high-risk subjects. Schizophr Res 2021;227:101-9.
61 Suor JH, Jimmy J, Monk CS, et al. Parsing differences in amygdala volume among individuals with and without social and generalized anxiety disorders across the lifespan. J Psychiatr Res 2020;128:83-9.

62 Chen Y, Cui Q, Fan Y-S, et al. Progressive brain structural alterations assessed via causal analysis in patients with generalized anxiety disorder. Neuropsychopharmacology 2020;45:1689-97.

63 Terlevic R, Isola M, Ragogna M, et al. Decreased hypothalamus volumes in generalized anxiety disorder but not in panic disorder. $J$ Affect Disord 2013;146:390-4.

$64 \mathrm{Li} \mathrm{H}$, Zhang B, Hu Q, et al. Altered heartbeat perception sensitivity associated with brain structural alterations in generalised anxiety disorder. Gen Psychiatr 2020;33:e100057.

65 Kunas SL, Hilbert K, Yang Y, et al. The modulating impact of cigarette smoking on brain structure in panic disorder: a voxel-based morphometry study. Soc Cogn Affect Neurosci 2020;15:849-59.

66 Wang X, Cheng B, Wang S, et al. Distinct grey matter volume alterations in adult patients with panic disorder and social anxiety disorder: a systematic review and voxel-based morphometry metaanalysis. J Affect Disord 2021;281:805-23.

67 Brooks SJ, Stein DJ. A systematic review of the neural bases of psychotherapy for anxiety and related disorders. Dialogues Clin Neurosci 2015;17:261-79.

68 Brühl AB, Delsignore A, Komossa K, et al. Neuroimaging in social anxiety disorder - a meta-analytic review resulting in a new neurofunctional model. Neurosci Biobehav Rev 2014;47:260-80.

$69 \mathrm{Li}$ J, Zhong Y, Ma Z, et al. Emotion reactivity-related brain network analysis in generalized anxiety disorder: a task fMRI study. BMC Psychiatry 2020;20:1-13

70 Yassa MA, Hazlett RL, Stark CEL, et al. Functional MRI of the amygdala and bed nucleus of the stria terminalis during conditions of uncertainty in generalized anxiety disorder. J Psychiatr Res 2012;46:1045-52.

71 Santos M, D'Amico D, Dierssen M. From neural to genetic substrates of panic disorder: insights from human and mouse studies. Eur J Pharmacol 2015;759:127-41.

72 Rosenbaum J. New directions in anxiety disorder treatment. Gen Psychiatr 2019;32:e100166.

73 Stuber GD, Sparta DR, Stamatakis AM, et al. Excitatory transmission from the amygdala to nucleus accumbens facilitates reward seeking. Nature 2011;475:377-80.

74 Heller AS, Cohen AO, Dreyfuss MFW, et al. Changes in corticosubcortical and subcortico-subcortical connectivity impact cognitive control to emotional cues across development. Soc Cogn Affect Neurosci 2016;11:nsw097.

75 Hare TA, Tottenham N, Galvan A, et al. Biological substrates of emotional reactivity and regulation in adolescence during an emotional go-nogo task. Biol Psychiatry 2008;63:927-34.

76 Casey BJ, Getz S, Galvan A. The adolescent brain. Dev Rev 2008;28:62-77.

77 Swartz JR, Carrasco M, Wiggins JL, et al. Age-Related changes in the structure and function of prefrontal cortex-amygdala circuitry in children and adolescents: a multi-modal imaging approach. Neuroimage 2014;86:212-20.

78 Lebel C, Beaulieu C. Longitudinal development of human brain wiring continues from childhood into adulthood. J Neurosci 2011;31:10937-47.

79 Phan KL, Orlichenko A, Boyd E, et al. Preliminary evidence of white matter abnormality in the uncinate fasciculus in generalized social anxiety disorder. Biol Psychiatry 2009;66:691-4.

80 Cunningham MG, Bhattacharyya S, Benes FM. Amygdalo-cortical sprouting continues into early adulthood: implications for the development of normal and abnormal function during adolescence. J Comp Neurol 2002;453:116-30.

81 Koss WA, Belden CE, Hristov AD, et al. Dendritic remodeling in the adolescent medial prefrontal cortex and the basolateral amygdala of male and female rats. Synapse 2014;68:61-72.

82 Meyer HC, Lee FS, Gee DG. The role of the endocannabinoid system and genetic variation in adolescent brain development. Neuropsychopharmacology 2018;43:21-33.

83 Gee DG, Humphreys KL, Flannery J, et al. A developmental shift from positive to negative connectivity in human amygdala-prefrontal circuitry. J Neurosci 2013;33:4584-93.

84 Silvers JA, Insel C, Powers A, et al. vIPFC-vmPFC-Amygdala interactions underlie age-related differences in cognitive regulation of emotion. Cereb Cortex 2017;27:3502-14.

85 Dong M, Xia L, Lu M, et al. A failed top-down control from the prefrontal cortex to the amygdala in generalized anxiety disorder: evidence from resting-state fMRI with Granger causality analysis. Neurosci Lett 2019;707:134314. 
86 Burghy CA, Stodola DE, Ruttle PL, et al. Developmental pathways to amygdala-prefrontal function and internalizing symptoms in adolescence. Nat Neurosci 2012;15:1736-41.

87 Wierenga L, Langen M, Ambrosino S, et al. Typical development of basal ganglia, hippocampus, amygdala and cerebellum from age 7 to 24. Neuroimage 2014;96:67-72.

88 Pattwell SS, Liston C, Jing D, et al. Dynamic changes in neural circuitry during adolescence are associated with persistent attenuation of fear memories. Nat Commun 2016;7:11475.

89 Bisby MA, Baker KD, Richardson R. Deficits in opioid receptormediated prediction error contribute to impaired fear extinction during adolescence. Behav Res Ther 2020;133:103713.

90 Sotres-Bayon F, Sierra-Mercado D, Pardilla-Delgado E, et al. Gating of fear in prelimbic cortex by hippocampal and amygdala inputs. Neuron 2012;76:804-12.

91 Clauss JA, Avery SN, Benningfield MM, et al. Social anxiety is associated with BNST response to unpredictability. Depress Anxiety 2019;36:666-75.

92 Saalfield J, Spear L. Fos activation patterns related to acute ethanol and conditioned taste aversion in adolescent and adult rats. Alcohol 2019;78:57-68.

93 Negrón-Oyarzo I, Pérez Miguel Ángel, Terreros G, et al. Effects of chronic stress in adolescence on learned fear, anxiety, and synaptic transmission in the rat prelimbic cortex. Behav Brain Res 2014;259:342-53.

94 Wu M, Mennin DS, Ly M, et al. When worry may be good for you: worry severity and limbic-prefrontal functional connectivity in latelife generalized anxiety disorder. J Affect Disord 2019;257:650-7.
95 Galvan A. Adolescent development of the reward system. Front Hum Neurosci 2010;4:6.

96 Lahat A, Benson BE, Pine DS, et al. Neural responses to reward in childhood: relations to early behavioral inhibition and social anxiety. Soc Cogn Affect Neurosci 2018;13:281-9.

97 Charpentier CJ, De Martino B, Sim AL, et al. Emotion-induced loss aversion and striatal-amygdala coupling in low-anxious individuals. Soc Cogn Affect Neurosci 2016;11:569-79.

98 DePasque S, Galván A. Frontostriatal development and probabilistic reinforcement learning during adolescence. Neurobiol Learn Mem 2017;143:1-7.

99 Liu W-jing, Yin D-zhi, Cheng W-hong, et al. Abnormal functional connectivity of the amygdala-based network in resting-state fMR in adolescents with generalized anxiety disorder. Med Sci Monit 2015;21:459-67.

100 Manning J, Reynolds G, Saygin ZM, et al. Altered resting-state functional connectivity of the frontal-striatal reward system in social anxiety disorder. PLoS One 2015;10:e0125286.

101 Sinclair D, Purves-Tyson TD, Allen KM, et al. Impacts of stress and sex hormones on dopamine neurotransmission in the adolescent brain. Psychopharmacology 2014;231:1581-99.

102 Li C, Liu Y, Yin S, et al. Long-term effects of early adolescent stress: dysregulation of hypothalamic-pituitary-adrenal axis and central corticotropin releasing factor receptor 1 expression in adult male rats. Behav Brain Res 2015;288:39-49.

103 Romeo RD. The impact of stress on the structure of the adolescent brain: implications for adolescent mental health. Brain Res 2017;1654:185-91.

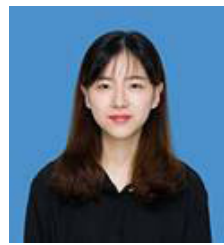

Shuqi Xie obtained a bachelor's degree in applied psychology from Guangzhou University of Chinese Medicine in China in 2019. She is currently a second year master's student at Laboratory of Psychological Health and Imaging in the Shanghai Mental Health Center, Shanghai Jiao Tong University School of Medicine, China. Her main research interest includes social anxiety disorder in adolescence. 